\title{
Exploratory Study of Epidermis, Basement Membrane Zone, Upper Dermis Alterations and Wnt Pathway Activation in Melasma Compared to Adjacent and Retroauricular Skin
}

\author{
Ana Cláudia Cavalcante Espósito, Gabrielli Brianezi ${ }^{1}$, Nathália Pereira de Souza ${ }^{1}$, \\ Luciane Donida Bartoli Miot, Hélio Amante Miot \\ Departments of Dermatology and Radioterapy, ${ }^{1}$ Patology, Botucatu School of Medicine, São Paulo State University, Botucatu, Brazil
}

\begin{abstract}
Background: Melasma is a chronic acquired focal hypermelanosis which pathogenesis has not been fully elucidated. Classical pathophysiologic studies have analysed the affected and perilesional areas, but little is known about the status of sun-protected skin, which is subjected to the same endogenous and genetic factors. Objective: To assess the histological characteristics of melasma compared to adjacent and retroauricular skin. Methods: Skin samples were collected from 10 female from: melasma, perilesional area and retroauricular. The samples were stained (haematoxylin-eosin, periodic acid-Schiff, Fontana-Masson, picrosirius red, toluidine blue and Verhoeff), immunolabelled for CD34 and Wnt1. The data from the skin sites were analysed simultaneously by a multivariate model. Results: Melasma skin exhibited noteworthy stratum corneum compaction, greater collagen heterogeneity, solar elastosis, higher number of mast cells, basement membrane zone (BMZ) damage, Wnt1 expression, pendulum melanocytes, higher cellularity and vascular proliferation at the superficial dermis. Stratum cor-
\end{abstract}

Received May 22, 2019, Revised November 17, 2019, Accepted for publication November 22, 2019

Corresponding author: Hélio Amante Miot, Department of Dermatology and Radioterapy, Botucatu School of Medicine, São Paulo State University, Botucatu 18618-000, Brazil. Tel: 55-14-38824922, Fax: 55-14-38824922, E-mail: heliomiot@fmb.unesp.br ORCID: https://orcid.org/0000-0002-2596-9294

This is an Open Access article distributed under the terms of the Creative Commons Attribution Non-Commercial License (http://creativecommons. org/licenses/by-nc/4.0) which permits unrestricted non-commercial use, distribution, and reproduction in any medium, provided the original work is properly cited.

Copyright (c) The Korean Dermatological Association and The Korean Society for Investigative Dermatology neum compaction, collagen heterogeneity and BMZ abnormalities were variables associated to melasma that not follow a continuum through retroauricular to adjacent skin. Mast cell count was the variable that disclosed correlation with the most other abnormalities as well as had the greater contribution in the multivariate model. Conclusion: In addition to melanocyte hyperactivity, melasma skin exhibits alterations in the epidermal barrier, upper dermis and BMZ, which differ from the adjacent sun-exposed skin and retroauricular skin, indicating a distinct phenotype, rather than a mere extension of photoageing or intrinsic ageing. Mast cells appear to play a central role in the physiopathology of melasma.

(Ann Dermatol 32(2) $101 \sim 108,2020$ )

-Keywords-

Basement membrane, Dermis, Epidermis, Melanosis

\section{INTRODUCTION}

Melasma is a chronic acquired focal hypermelanosis that affects sun-exposed areas, particularly the face, in female. The locations of melasma in visible areas, the young age of patients and the frequent relapse (even during treatment) result in a considerable impact on the quality of life $^{1}$. Facial melasma is independently associated with miscegenation and the high occurrence within familiars $(40 \% \sim 60 \%)$ suggests a genetic predisposition, which is best explicated by a dominant pattern of segregation ${ }^{2,3}$.

Although its pathogenesis has not yet been fully elucidated, it has already been established that melasma is not due only to melanocyte hypertrophy but it results from a 
global alteration of the full epidermal-melanin unit, fibroblast and endothelium, with significant abnormalities in the upper dermis and basement membrane zone (BMZ) $)^{4,5}$. To date, no other previous research have explored all these alterations together and analysed the correlations of these variables. Moreover, the classic studies on the histology of melasma merely evaluated the affected skin and the adjacent area, and little is known about the behaviour of sun-protected skin, which is still subjected to the same endogenous and genetic factors as melasma skin.

The aims of the present study were to assess the histological characteristics of melasma skin with an integrative view and investigate the possible correlation between morphological abnormalities and epidermal expression of Wnt1 in affected skin, perilesional and retroauricular skin.

\section{MATERIALS AND METHODS}

Botucatu School of Medicine, São Paulo State University institutional committee of ethics on research approved the project (no. 1,602,185). A cross-sectional study with intrasubject control was performed, in which were includeed 10 adult female ( $>18$ years old) with facial melasma who had not been treated in the past 30 days or more, except for use of sunscreen. The participants were selected using convenience sampling from January through June-2016. Patients with systemic diseases and those who were pregnant or using anticoagulants were not included. The study design for 10 patients (30 samples) was based on the expectative of more than $50 \%$ of difference between the topographies (dependent samples), with $\alpha=0.05$ and $\beta=0.2^{6}$. Skin samples (3-mm punch) were taken from three different sites: facial melasma, adjacent healthy skin (up to 2 $\mathrm{cm}$ from melasma skin) and retroauricular skin (which is a relatively non sun-exposed skin and with a pattern of melanocytes distribution similarly to the face).

The samples were processed for histochemical staining techniques (haematoxylin-eosin, Fontana-Masson, periodic acid-Schiff [PAS], picrosirius red, Verhoeff and toluidine blue), for immunohistochemical (goat polyclonal anti-CD 34 primary antibody, C-18, sc-7045; Santa Cruz Biotechnology, Dallas, TX, USA) and for double-labelling direct immunofluorescence (Wnt1), which objective was to analyse the anatomical differences between sites, degree of solar elastosis, amount of epidermal and dermal melanin, BMZ abnormalities, elastic and collagen fibres, pendulum melanocytes, number of mast cells and dermal cellularity, vascular components and expression of Wnt pathway. The stratum corneum compaction and solar elastosis were analyzed through a qualitative method (for compaction: 0, no compaction; 1 , moderate, and 2, severe compaction; for solar elastosis: 0 , absent; 1 , moderate, and 2, severe solar elastosis).

The BMZ damages were counted in PAS stain (mean count per high-power field: $\times 400$ ), totalizing approximately 13 fields in each histologic fragment. Collagen homogeneity was evaluated in picrosirius red using the box-counting fractal dimension to quantify the organization of collagen fibers. It results how thick a set occupies the metric space in which it exists.

The protocol employed in the Wnt included slide deparaffinisation, antigen retrieval in citrate solution and heating cycles in a microwave oven. Non-specific protein blocking was performed with $1 \%$ milk. Histological sections were incubated with primary antibody-anti-Wnt1 antibody (ab15251; Abcam, Cambridge, MA, USA) 1:100 overnight in a wet chamber at $4^{\circ} \mathrm{C}$. Following rinsing, the sections were incubated with secondary antibody conjugated to Alexa Fluor $^{\mathbb{R}} 594$ dye (Thermo Fisher Scientific, Waltham, MA, USA) 1:500 for 20 minutes in a wet chamber at $37^{\circ} \mathrm{C}$ in a dark room. Counterstaining with 4',6-diamidino-2-phenylindole was performed for nuclear labelling.

Photographs were taken in triplicate from regular interfollicular, artefact-free areas from each histological section using a Coolscope II microscope (Nikon Inc., Tokyo, Japan), resulting in tagged image file format images with $1280 \times 1024$ pixels (24-bit colour). The intensity of epidermal labelling (excluding the stratum corneum in the instances with autofluorescence) was analysed by two different dermatologists with wide knowledge in dermatopathology, using the software Image 1.51 e $(0-255$ grey hue variation) for the various colour channels ${ }^{7}$. The sites of the samples were blinded during the assessment.

The data were compared among skin types using a generalised linear mixed effects model, with a robust covariance structure, a probability distribution adjusted for each sample (normal or gamma) and post hoc analysis using a sequential Sidak algorithm ${ }^{8}$.

The correlation of variables that were significant on bivariate analysis was assessed using Spearman's test and then included in an exploratory multiple correspondence analysis model ${ }^{9,10}$.

The analyses were performed using IBM SPSS ver. 25.0 software (IBM Corp., Armonk, NY, USA); the significance level was set as $p \leq 0.05$.

\section{RESULTS}

The mean age (standard deviation) of the participants was 42.9 (8.9) years, the length of melasma was 16.7 (7.9) years, and all cases exhibited phototypes III or IV.

The main results of immunologic and histomorphometric 
Epidermal and Upper Dermis Abnormalities in Melasma

Table 1. Immunological and histomorphometric measurements related to patients with melasma per investigated skin site

\begin{tabular}{|c|c|c|c|c|}
\hline Histomorphometric and immunological measurement & Melasma skin & Perilesional skin & Retroauricular skin & $p$-value \\
\hline \multicolumn{5}{|l|}{ Haematoxylin-eosin } \\
\hline Stratum corneum thickness (micra) & $13.4(4.2)$ & $13.3(3.7)$ & $18.1(5.3)^{\S, \|}$ & $<0.01^{9}$ \\
\hline Viable epithelium thickness (micra) & $58.8(9.7)$ & $55.2(10.9)$ & $53.1(9.6)$ & 0.59 \\
\hline Total epidermis thickness (micra) & $72.1(11.0)$ & $69.6(15.1)$ & $71.2(11.9)$ & 0.91 \\
\hline Stratum corneum compaction (qualitative) ${ }^{*, \dagger}$ & $1(1 \sim 2)$ & $1(0 \sim 1)^{\S}$ & $0(0)^{\S, \|}$ & $<0.01^{9}$ \\
\hline Solar elastosis (qualitative) ${ }^{*, \neq}$ & $1(1 \sim 2)$ & $0(0 \sim 1)^{\S}$ & $0(0)^{\S, \|}$ & $<0.01^{9}$ \\
\hline Cellularity of the upper dermis (per $\times 40$ field) & $53.6(7.5)$ & $45.4(6.2)^{\S}$ & $37.7(6.6)^{\S, \|}$ & $<0.01^{9}$ \\
\hline \multicolumn{5}{|l|}{ Fontana-Masson } \\
\hline Melanin density in epidermis & $0.35(0.06)$ & $0.32(0.08)^{\S}$ & $0.25(0.08)^{\S, \|}$ & $<0.01^{9}$ \\
\hline Melanin density in dermis $(\times 100)^{*}$ & $0.19(0.06 \sim 0.75)$ & $0.25(0.20 \sim 0.35)$ & $0.25(0.16 \sim 0.45)$ & 0.82 \\
\hline \multicolumn{5}{|l|}{ Periodic acid-Schiff } \\
\hline Basement membrane lesions (per $\times 40$ field) & $2.1(0.6)$ & $1.7(0.8)^{\S}$ & $1.0(0.5)^{\S, \|}$ & $<0.01^{9}$ \\
\hline Pendulum melanocytes (per $\times 40$ field $)^{*}$ & $2.7(2.7 \sim 3.3)$ & $1.3(1.3 \sim 3.0)^{\S}$ & $1.3(1.0 \sim 1.7)^{\S}$ & $<0.01^{9}$ \\
\hline \multicolumn{5}{|l|}{ Verhoeff } \\
\hline Elastotic material density in upper dermis & $0.29(0.07)$ & $0.25(0.04)^{\S}$ & $0.19(0.04)^{\S, \|}$ & $<0.01^{9}$ \\
\hline \multicolumn{5}{|l|}{ Picrossirius red } \\
\hline Collagen density in upper dermis & $0.63(0.09)$ & $0.68(0.07)$ & $0.66(0.06)$ & 0.15 \\
\hline Collagen homogeneity (fractal dimension) & $1.53(0.02)$ & $1.56(0.04)^{\S}$ & $1.57(0.03)^{\S}$ & $<0.01^{9}$ \\
\hline \multicolumn{5}{|l|}{ Toluidine blue } \\
\hline Mast cell $($ per $\times 40$ field $)$ & $6.4(1.5)$ & $3.6(0.7)^{\S}$ & $2.5(0.8)^{\S, \|}$ & $<0.01^{9}$ \\
\hline \multicolumn{5}{|l|}{ CD34 } \\
\hline Blood vessels density in the upper dermis & $6.8(1.4)$ & $4.3(0.8)^{\S}$ & $4.5(1.5)^{\S}$ & $<0.01^{9}$ \\
\hline Wnt1 (epidermal pixel intensity) & $67(29)$ & $54(30)^{\S}$ & $57(32)^{\|}$ & 0.02 \\
\hline
\end{tabular}

Values are presented as mean \pm standard deviation.

${ }^{*}$ Median (p25-p75). ${ }^{\dagger} 0$ : no corneal compaction; 1 : mild to moderate corneal compaction, 2: significant corneal compaction. ${ }^{\ddagger} 0$ : absence of solar elastosis; 1 : mild to moderate solar elastosis, 2 : intense solar elastosis. ${ }^{\S} p<0.05$ in relation to melisma, $" p<0.05$ in relation to adjacent skin. ${ }^{\mathrm{p}} p<0.05$.

analysis are disclosed in Table 1. Thinning of the stratum corneum in the most sun-exposed fragments (Fig. 1) and its greater compaction in the melasma lesions were remarkable. Progressive melanin pigmentation of the epidermis was detected across the skin topographies (Fig. 1). Solar elastosis was more prominent in the most sun-exposed skin, particularly the melasma lesions (Fig. 1, 2). Similarly, the dermal collagen was more heterogeneous in the papillary dermis of melasma skin than in the other sites (Fig. 2).

Pendulum melanocytes (Fig. 3) were more frequent in melasma and sun-exposed skin, as the dermal cellularity was higher, and there was a larger number (and diameter) of vascular structures (Fig. 3), mast cells (Fig. 4), and epidermal Wnt1 (Fig. 5) in melasma.

The correlations among histological variables are described in Table 2. The compaction of the stratum corneum, cellularity of the superficial dermis, mast cell density and solar elastosis were the variables that exhibited the strongest patterns of correlation. Mast cell count was the variable that disclosed correlation with the most other abnormalities.
The perceptual map for the multiple correspondence analysis (Fig. 6) included 13 variables and accounted for $65 \%$ of the variance of the two-component model. The topographies were well characterized by the variables. Solar elastosis, collagen heterogeneity, dermal cellularity, mast cell number, presence of pendulum melanocytes, vascular structures, abnormalities of the elastic fibres and Wnt1 expression shown the strongest correlation with the facial skin topographies. Melasma exhibited preferential association with mast cell number, superficial dermal cellularity and abnormalities of the elastic fibres. Mast cell count was the variable that disclosed greater inertia from the origin, in the three quadrants, providing the greater contribution to the model. Stratum corneum compaction, density of epidermal melanin, collagen heterogeneity and BMZ abnormalities were variables associated to melasma that not follow a continuum through retroauricular to adjacent skin.

\section{DISCUSSION}

Histological differences and $\mathrm{Wnt} / \beta$-catenin activation were explored among facial melasma, perilesional and retro- 
auricular skin. The comparison of these three areas provides a model that allows inferences about the role that environmental stimuli (e.g., ultraviolet radiation [UVR]) and intrinsic factors, such as genetic and hormonal influences, play in the pathogenesis of melasma ${ }^{11}$.
Stratum corneum compaction together with functional lipid metabolism disorder are associated to the functional integrity of the skin barrier. The difference in its compaction between melasma and perilesional skin (both sun-exposed areas), favours the hypothesis that melasma is associated
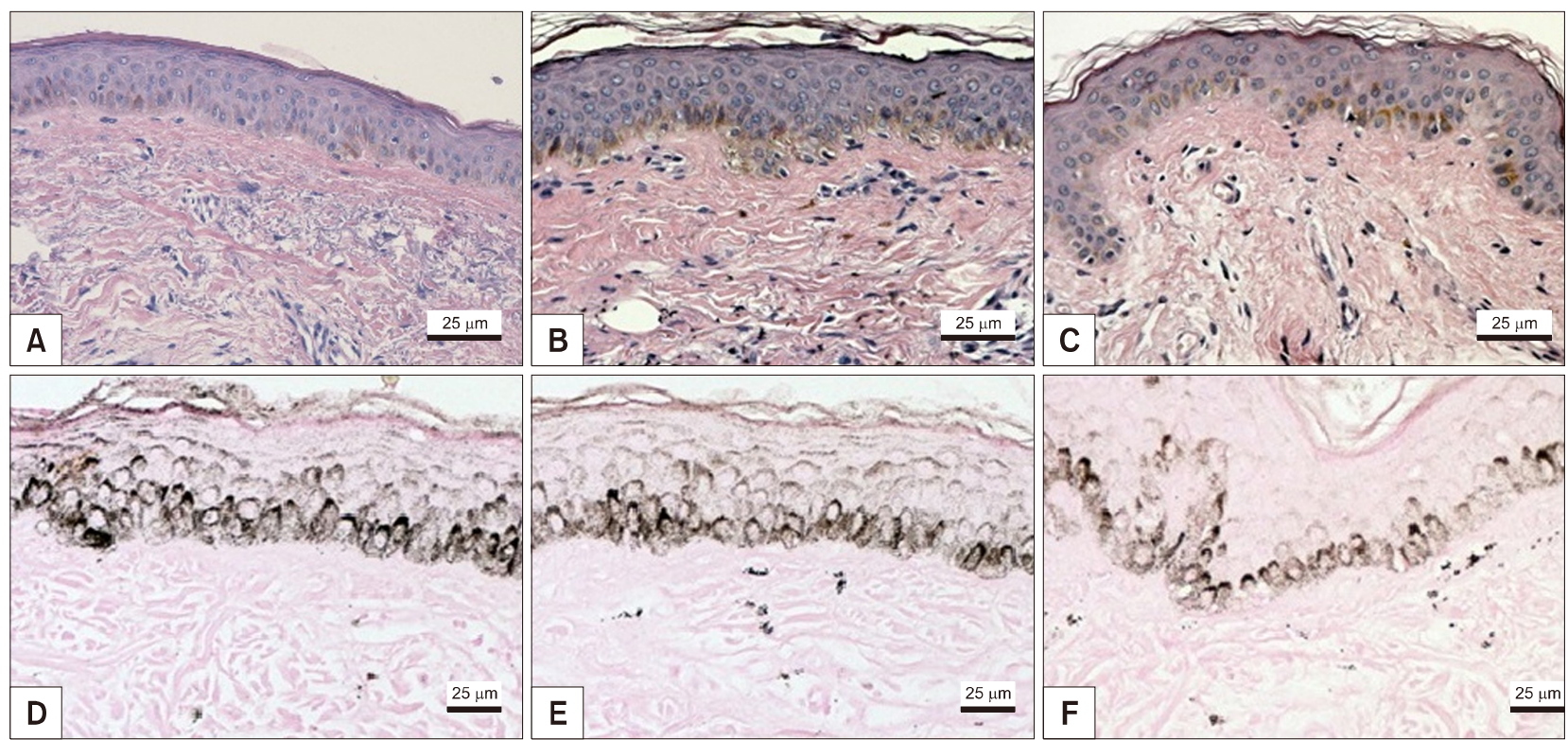

Fig. 1. Haematoxylin-eosin staining shows thinning of the stratum corneum in melasma (A) and adjacent (B) skin and greater compaction in the melasma sample. Solar elastosis exhibited increased progressively expression across the investigated skin types and it was greater in melasma (A) than in the adjacent skin (B) and retroauricular (C) skin. Fontana-Masson staining shows the progressive increasing in the epidermal melanin pigmentation across the skin types, which was greater in melasma (D) than in the adjacent skin (E) and retroauricular $(F)$ skin. The melanin density in the dermis did not differ between samples. $(A \sim C) \times 100,(D \sim E) \times 200$.
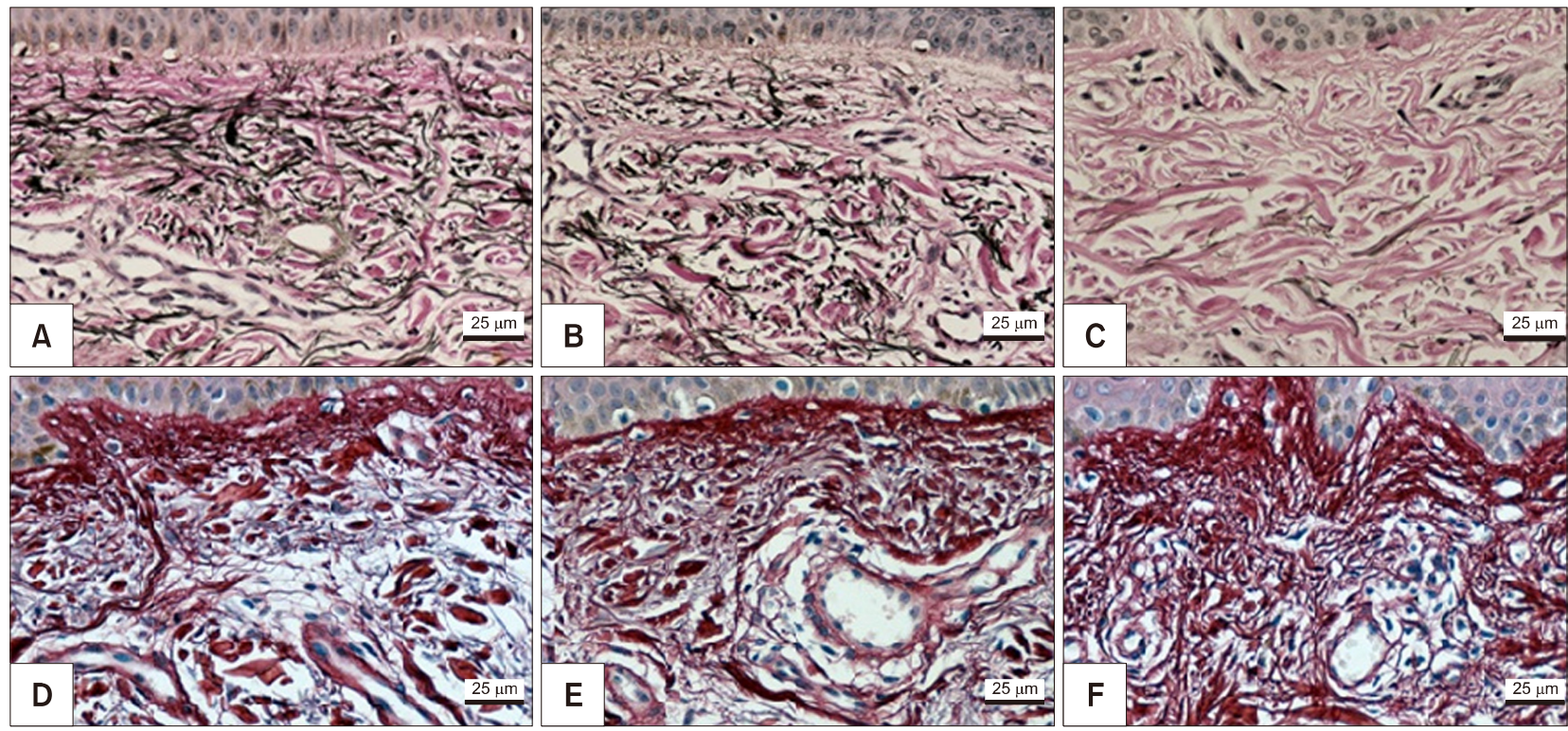

Fig. 2. Melasma (A), adjacent (B) and retroauricular (C) skin subjected to Verhoeff staining: the elastotic material is more prominent in the most sun-exposed skin, specifically melasma (A). Picrosirius red staining showed greater collagen heterogeneity in the papillary dermis of melasma (D) than in the adjacent $(E)$ and retroauricular $(F)$ skin. $(A \sim E) \times 200$. 

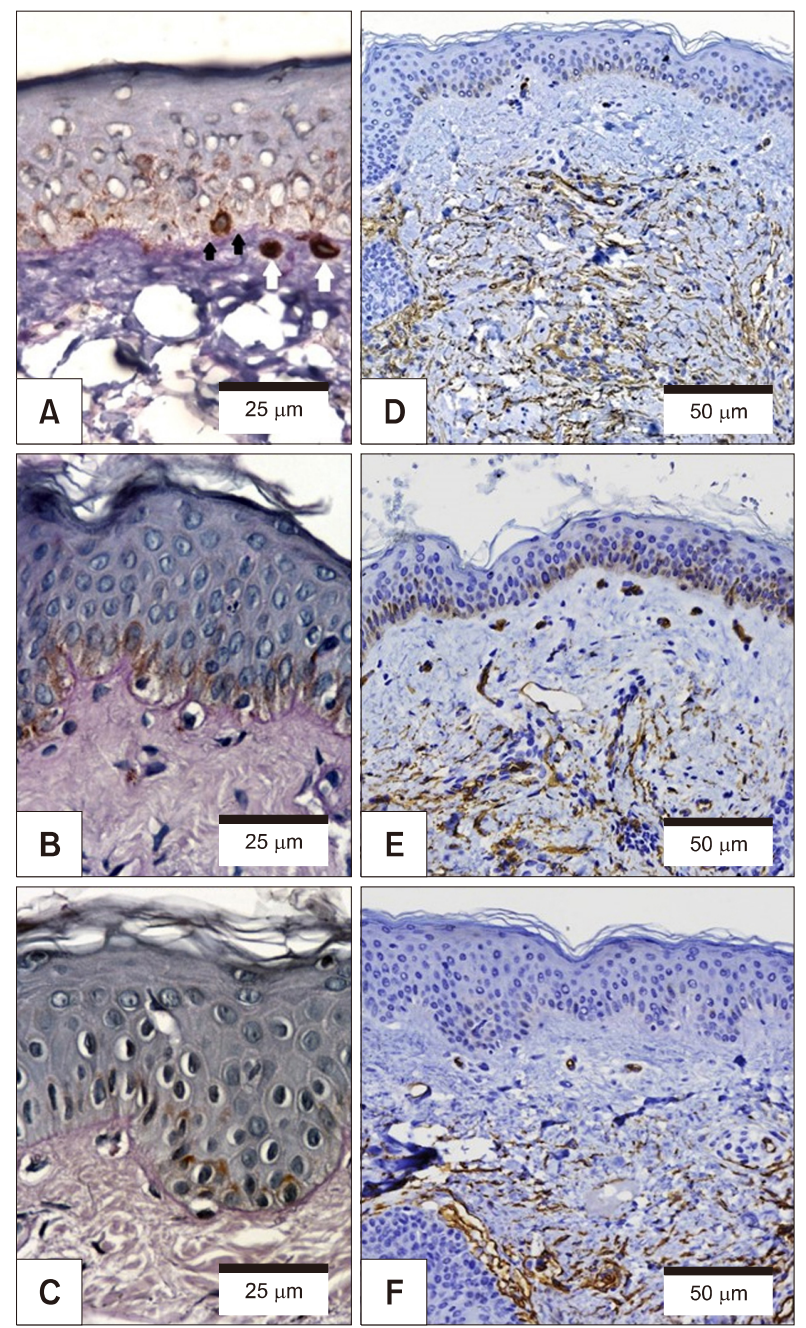

Fig. 3. Melasma (A), adjacent (B) and retroauricular (C) skin subjected to periodic acid-Schiff staining: basement membrane changes was more prominent in melasma skin (A). CD34 immunohistochemical showed larger number of blood vessels more evident in melasma (D) than in the adjacent (E) and retroauricular (F) skin. The white arrows are pointing to the pendulum melanocytes while the black arrows are pointing the basement membrane disruptions. $(A \sim C) \times 100,(D \sim E) \times 200$.

with greater damage to the skin barrier ${ }^{12,13}$.

Melanogenesis in melasma involves epithelial secretion of $\alpha$-melanocyte stimulating hormone ( $\alpha \mathrm{bMSH}$ ) and activation of the Wnt pathway; nonetheless, it seems to be independent of the stimulation by ultraviolet radiation/p53, interleukin (IL)- $1 \alpha$, cyclooxygenase-2/prostaglandin E2, WIF-1 and ASIP ${ }^{14}$. The melanin synthesis in melasma suggests an microenvironment that favours focal melanogenesis in an area exposed to similar UVR to the adjacent skin. In this study, the melanin density in the epidermis exhibited a correlation with the number of cells in the upper dermis, the amount of vascular structures and mast

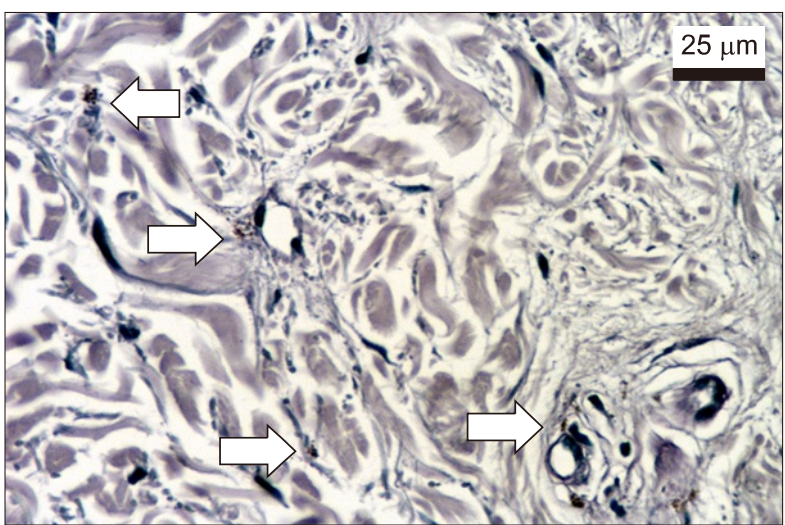

Fig. 4. Mast cells in the superficial dermis (arrows) of melasma skin, with preferential perivascular topography and metachromatic granules (purple), as assessed using toluidine blue staining $(\times 100)$.

cells, which suggests that dermal stimuli play a major role in the melanogenesis of melasma.

The number of pendulum melanocytes exhibited significant progression as a function of the assessed skin area. The density of pendulum melanocytes was correlated with stratum corneum compaction, solar elastosis, counting of mast cells and abnormalities of the elastotic material but was not correlated with the number of BMZ failures. Experimental exposure of photoprotected skin to UVA triggers abnormalities similar to those found in melasma ${ }^{15}$. Beyond the evident epidermal abnormalities, there was a significant difference in the degree of elastosis between melasma and the adjacent skin, one might hypothesise that this change is not just a secondary epiphenomenon of photoageing but a condition relevant to the development of melasma. Solar elastosis was correlated with collagen heterogeneity, superficial dermis cellularity, counting of mast cells and dermal blood vessels, pendulum melanocytes, stratum corneum compaction and elastic fibre damage, which suggests an integrative role of the various components in the physiopathology of melasma.

Collagen heterogeneity, which was assessed through fractal dimension, reflects greater fragmentation of collagen fibers. Its correlation with solar elastosis and mast cell count in upper dermis suggests the role of ultraviolet radiation and inflammatory mediators in collagen alterations, as well as it occurs in photoaging, where the dermis disruption is clinically manifested by increased sagging and wrinkled skin.

The increased dermal cellularity in melasma lesions might be due to inflammation and oxidative damage ${ }^{11}$. UVB radiation is able to induce the secretion of cytokines, such as endothelin-1, IL-1, nitric oxide, stem cell factor and $\alpha \mathrm{MSH}$, 

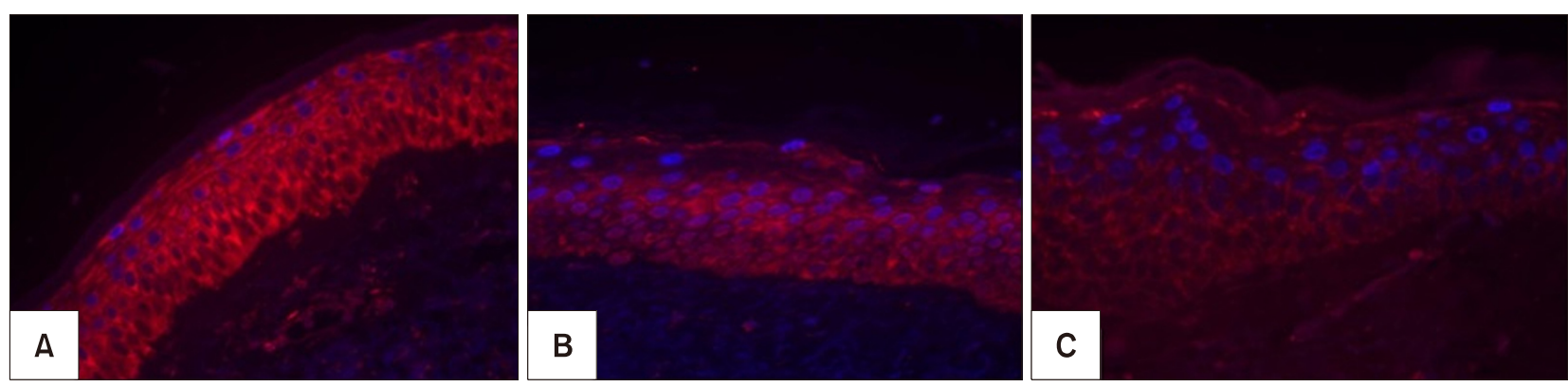

Fig. 5. Melasma (A), adjacent healthy skin (B) and retroauricular skin (C) with double-labelling double immunofluorescence for Wnt1 (red/cytoplasmic). The blue colour is related to the nucleus marker (4',6-diamidino-2-phenylindole).

Table 2. Correlation (Spearman's rho) of histomorphometric measurements regarding the patients with melasma

\begin{tabular}{|c|c|c|c|c|c|c|c|c|c|c|c|}
\hline $\begin{array}{l}\text { Histomorphometric } \\
\text { measurement }\end{array}$ & Corn & $\begin{array}{l}\text { Compact- } \\
\text { Corn }\end{array}$ & Verh & Elastos & CollHomog & DermCell & BasMemb & Masto & VasCD34 & Pendul & Wnt1 \\
\hline MelEpid & $-0.36^{*}$ & 0.19 & -0.05 & 0.23 & -0.16 & $0.41 *$ & 0.25 & $0.37^{*}$ & $0.45^{*}$ & 0.04 & -0.23 \\
\hline Corn & & -0.31 & -0.11 & -0.23 & 0.02 & $-0.38^{*}$ & $-0.36^{*}$ & -0.15 & -0.04 & -0.24 & -0.07 \\
\hline CompactCorn & & & $0.60 *$ & $0.57^{*}$ & -0.16 & $0.58 *$ & 0.13 & $0.51 *$ & 0.15 & $0.60^{*}$ & 0.18 \\
\hline Verh & & & & $0.54 *$ & -0.27 & 0.27 & -0.15 & $0.46^{*}$ & 0.11 & $0.53^{*}$ & -0.06 \\
\hline Elastos & & & & & $-0.39^{*}$ & $0.43^{*}$ & 0.17 & $0.66^{*}$ & $0.41 *$ & $0.37^{*}$ & 0.30 \\
\hline CollHomog & & & & & & -0.30 & -0.10 & $-0.47^{*}$ & -0.20 & -0.18 & 0.00 \\
\hline DermCell & & & & & & & $0.37 *$ & $0.70 *$ & 0.27 & 0.30 & 0.32 \\
\hline BasMemb & & & & & & & & 0.29 & 0.27 & 0.11 & 0.24 \\
\hline Masto & & & & & & & & & $0.45^{*}$ & $0.49 *$ & $0.40 *$ \\
\hline VasCD34 & & & & & & & & & & 0.26 & -0.07 \\
\hline Pend & & & & & & & & & & & 0.09 \\
\hline
\end{tabular}

Corn: stratum corneum thickness, CompactCorn: stratum corneum compaction, Verh: assessment of elastic fibres by means of Verhoeft staining, Elastos: solar elastosis, DermCell: superficial dermis cellularity, VasCD34: density of blood vessels in the superficial dermis, Pendul: pendulum melanocytes, Wnt: density of anti-Wnt antibody labelling in the epidermis, MelEpid: melanin in the epidermis, BasMemb: density of basement membrane lesions, Masto: mast cell density in the superficial dermis, CollHomog: collagen heterogeneity in the upper dermis. ${ }^{*} p \leq 0.05$.

which stimulate melanocyte mitosis or melanogenesis ${ }^{11,16}$. The density of mast cells increases according to the degree of photodamage in sun-exposed areas ${ }^{11}$. Histamine stimulates proliferation and migration of melanocytes through activation of protein kinase-A via the $\mathrm{H} 2$ receptor, thus behaving as a direct stimulator of melanogenesis ${ }^{16,17}$. Mast cells also secret paracrine mediators factors that induce elastin production by fibroblasts ${ }^{18}$. In addition, mast cells produce tryptase, which participate in the damage to the extracellular matrix and basement membrane, (through matrix metallopeptidases 9-collagen IV damage), what can influence the occurrence of collagen heterogeneity and pendulum melanocytes, which were correlated with the presence of mast cells in the present study ${ }^{19,20}$. Our results suggest mast cells as playing a central role in the pathophysiology of melasma, and therapeutic trials with mastocyte stabilizers drugs are warranted.

The melasma samples exhibited a larger number of vascular structures, which was correlated with solar elastosis, the presence of mast cells and epidermal pigmentation. Angiogenesis might be stimulated by UVR, fibroblast growth factor secretion, IL-8 and vascular endothelial growth factor (VEGF) ${ }^{21,22}$. Studies are needed to demonstrate the direct action of VEGF in the melanogenesis in melasma $^{4,23}$. Endothelin-1, which is produced by endothelial and inflammatory cells, promotes the upregulation of key genes for melanogenesis, such as the genes encoding tyrosinase and the microphthalmia-associated transcription factor. The endothelium-induced melanogenic stimulus is even stronger after exposure to UVR, which reinforces its role in the pathophysiology of melasma ${ }^{24}$.

The $\mathrm{Wnt} / \beta$-catenin pathway includes a large family of proteins with different cell functions, such as the melanoblasts migration, proliferation and induction of pigmentation $^{25}$. Wnt1 is a ligand of transmembrane frizzled receptors, which promote de accumulation and stabilization of $\beta$-catenin ${ }^{26}$. Wnt1 was select to be analysed because it is the mainly activator of $\beta$-catenin pathway. The dynamic 


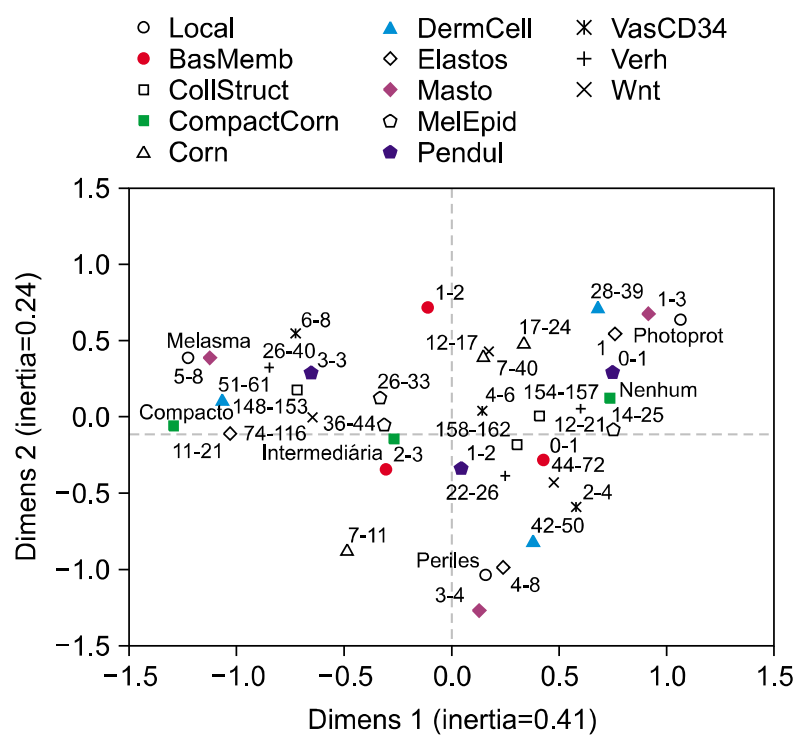

Fig. 6. Perceptual map from multiple correspondence analysis of the investigated histological variables for melasma, adjacent skin and retroauricular skin. Thirteen variables were included allowing to explain $65 \%$ of the variance of the two-component model. Solar elastosis, collagen heterogeneity, dermal cellularity, mast cell number, presence of pendulum melanocytes, presence of vascular structures, abnormalities of the elastic fibres and Wnt1 expression shown the strongest correlation with the facial skin locations because they present similar quadrangular distribution.

of this pathway seems to be altered in patients with melasma. The correlation between mast cells and $\mathrm{Wnt}$ in the skin seems to result from the inflammatory microenvironment and chronic upper dermal and BMZ damage/repair process that occur in melasma, triggering inadequate and long-lasting activation of $\mathrm{Wnt} / \beta$-catenin signalling ${ }^{27}$.

The present study exhibits potential limitations as the sampling of facial melasma from only one center in Brazil. This condition does not allow generalising the results to other population groups, males or extra-facial melasma. The use of retroauricular skin as a model of photoprotection is not free from criticism, although this skin lends itself to comparison with perilesional skin as part of a sun-exposure continuum. Lastly, the modest sample size, despite attaining adequate statistical power, did not allow for further subgroup analysis, nonetheless it did not hinder for to achieve significance.

In addition to melanocyte hypertrophy, melasma skin exhibits concomitant abnormalities in the epidermal barrier, upper dermis, BMZ and $\mathrm{Wnt} / \beta$-catenin pathway activation that differ from those of adjacent sun-exposed and retroauricular skin and thus form a distinct phenotype rather than a mere extension of photoageing or intrinsic ageing, as well as a completely distinct pathogenesis from other melanogenic disorders, as solar lentigo, ephelides, post-inflammatory hyperpigmentation or mastocytosis.

\section{ACKNOWLEDGMENT}

CNPq - 401309/2016-9 and FUNADERSP for funding.

\section{CONFLICTS OF INTEREST}

The authors have nothing to disclose.

\section{ORCID}

Ana Cláudia Cavalcante Espósito, https://orcid.org/00000001-9283-2354

Gabrielli Brianezi, https://orcid.org/0000-0003-3482-0296

Nathália Pereira de Souza, https://orcid.org/0000-0001-60520356

Luciane Donida Bartoli Miot, https://orcid.org/0000-00022388-7842

Hélio Amante Miot, https://orcid.org/0000-0002-2596-9294

\section{REFERENCES}

1. Maranzatto CF, Miot HA, Miot LD, Meneguin S. Psychometrican analysis and dimensional structure of the Brazilian version of melasma quality of life scale (MELASQoL-BP). An Bras Dermatol 2016;91:422-428.

2. Holmo NF, Ramos GB, Salomão H, Werneck RI, Mira MT, Miot LDB, et al. Complex segregation analysis of facial melasma in Brazil: evidence for a genetic susceptibility with a dominant pattern of segregation. Arch Dermatol Res 2018;310:827-831.

3. D'Elia MP, Brandão MC, de Andrade Ramos BR, da Silva MG, Miot LD, Dos Santos SE, et al. African ancestry is associated with facial melasma in women: a cross-sectional study. BMC Med Genet 2017;18:17.

4. Kwon SH, Hwang YJ, Lee SK, Park KC. Heterogeneous pathology of melasma and its clinical implications. Int J Mol Sci 2016;17:E824.

5. Brianezi G, Handel AC, Schmitt JV, Miot LD, Miot HA. Changes in nuclear morphology and chromatin texture of basal keratinocytes in melasma. J Eur Acad Dermatol Venereol 2015;29:809-812.

6. Miot HA. Sample size in clinical and experimental trials. J Vasc Bras 2011;10:275-278.

7. Schneider CA, Rasband WS, Eliceiri KW. NIH Image to ImageJ: 25 years of image analysis. Nat Methods 2012;9:671675.

8. Miot HA. Assessing normality of data in clinical and experimental trials. J Vasc Bras 2017;16:88-91.

9. Sourial N, Wolfson C, Zhu B, Quail J, Fletcher J, Karunananthan $\mathrm{S}$, et al. Correspondence analysis is a useful tool to 
uncover the relationships among categorical variables. J Clin Epidemiol 2010;63:638-646.

10. Miot HA. Correlation analysis in clinical and experimental studies. J Vasc Bras 2018;17:275-279.

11. Miot LD, Miot HA, Polettini J, Silva MG, Marques ME. Morphologic changes and the expression of alpha-melanocyte stimulating hormone and melanocortin-1 receptor in melasma lesions: a comparative study. Am J Dermatopathol 2010;32:676-682.

12. Sarkar R, Arora P, Garg VK, Sonthalia S, Gokhale N. Melasma update. Indian Dermatol Online J 2014;5:426435.

13. Bhawan J, Andersen W, Lee J, Labadie R, Solares G. Photoaging versus intrinsic aging: a morphologic assessment of facial skin. J Cutan Pathol 1995;22:154-159.

14. Espósito ACC, Brianezi G, de Souza NP, Miot LDB, Marques MEA, Miot HA. Exploring pathways for sustained melanogenesis in facial melasma: an immunofluorescence study. Int J Cosmet Sci 2018;40:420-424.

15. Bacharach-Buhles $M$, Lubowietzki $M$, Altmeyer P. Dosedependent shift of apoptotic and unaltered melanocytes into the dermis after irradiation with UVA 1. Dermatology 1999;198:5-10.

16. Imokawa G, Yada Y, Morisaki N, Kimura M. Biological characterization of human fibroblast-derived mitogenic factors for human melanocytes. Biochem J 1998;330(Pt 3): 1235-1239.

17. Yoshida M, Takahashi $Y$, Inoue S. Histamine induces melanogenesis and morphologic changes by protein kinase A activation via $\mathrm{H} 2$ receptors in human normal melanocytes. J Invest Dermatol 2000;114:334-342.

18. Hernández-Barrera R, Torres-Alvarez B, Castanedo-Cazares JP, Oros-Ovalle C, Moncada B. Solar elastosis and presence of mast cells as key features in the pathogenesis of melasma. Clin Exp Dermatol 2008;33:305-308.
19. Iddamalgoda A, Le QT, Ito K, Tanaka K, Kojima H, Kido H. Mast cell tryptase and photoaging: possible involvement in the degradation of extra cellular matrix and basement membrane proteins. Arch Dermatol Res 2008;300 Suppl 1:S69-S76.

20. Espósito ACC, Brianezi G, de Souza NP, Santos DC, Miot LDB, Miot HA. Ultrastructural characterization of damage in the basement membrane of facial melasma. Arch Dermatol Res 2019. doi: 10.1007/s00403-019-01979-w. [Epub ahead of print]

21. Krämer M, Sachsenmaier $C$, Herrlich $P$, Rahmsdorf HJ. UV irradiation-induced interleukin-1 and basic fibroblast growth factor synthesis and release mediate part of the UV response. J Biol Chem 1993;268:6734-6741.

22. Kim EH, Kim YC, Lee ES, Kang HY. The vascular characteristics of melasma. J Dermatol Sci 2007;46:111-116.

23. Kim EJ, Park HY, Yaar M, Gilchrest BA. Modulation of vascular endothelial growth factor receptors in melanocytes. Exp Dermatol 2005;14:625-633.

24. Regazzetti C, De Donatis GM, Ghorbel HH, Cardot-Leccia N, Ambrosetti D, Bahadoran P, et al. Endothelial Cells promote pigmentation through endothelin receptor $\mathrm{B}$ activation. J Invest Dermatol 2015;135:3096-3104.

25. Whyte JL, Smith AA, Helms JA. Wnt signaling and injury repair. Cold Spring Harb Perspect Biol 2012;4:a008078.

26. Dijksterhuis JP, Petersen J, Schulte G. WNT/Frizzled signalling: receptor-ligand selectivity with focus on FZD-G protein signalling and its physiological relevance: IUPHAR Review 3. Br J Pharmacol 2014;171:1195-1209.

27. Yamada $T$, Hasegawa $S$, Inoue $Y$, Date $Y$, Yamamoto $N$, Mizutani $\mathrm{H}$, et al. Wnt/ $\beta$-catenin and kit signaling sequentially regulate melanocyte stem cell differentiation in UVBinduced epidermal pigmentation. J Invest Dermatol 2013; 133:2753-2762. 